\title{
Identification of an Anopheles Lineage-Specific Unique Heme Peroxidase HPX15: A Plausible Candidate for Arresting Malaria Parasite Development
}

Mithilesh Kajla ${ }^{1}$, Kuldeep Gupta ${ }^{1}$, Parik Kakani ${ }^{1}$, Rini Dhawan ${ }^{1}$, Tania Pal Choudhury ${ }^{1}$, Lalita Gupta ${ }^{1}$, Surendra K Gakhar ${ }^{2}$ and Sanjeev Kumar $^{1 *}$

${ }^{1}$ Molecular Parasitology and Vector Biology Laboratory, Department of Biological Sciences, Birla Institute of Technology and Sciences, Pilani (BITS-Pilani), Pilani-333 031, Rajasthan, India

${ }^{2}$ Department of Life Sciences, Maharashi Dayanand University, Rohtak, Haryana, India

\begin{abstract}
Background: Human malaria parasite Plasmodium falciparum is transmitted by several species of Anopheles mosquito. The advancement of drug-resistant parasites and insecticide resistance in mosquito vectors are major hurdles in the malaria control. Alternatively, the manipulation of mosquito immunity is also an ideal way to block Plasmodium development inside the insect host. This approach demands the identification of key mosquito molecules that regulate anti-plasmodial immunity. Our previous findings revealed that the silencing of Anopheles gambiae heme peroxidase 15 (AgHPX15, AGAP013327) induced mosquito innate immunity and drastically suppressed the development of human and rodent malaria parasites. Further, we aim to characterize HPX15 orthologs in Indian malaria vectors and other worldwide-distributed anophelines to understand the novelty of this molecule as a plausible target to block Plasmodium development.
\end{abstract}

Method: AgHPX15 orthologs were cloned from major Indian malaria vectors $A$. stephensi and A. culicifacies and their conserve domains were determined by CDD search tool. The sequence homology and phylogenetic relationship of these clones with other heme peroxidases was analysed using Mega 5.2 software.

Results and conclusion: We found that $A$. stephensi AsHPX15 and A. culicifacies AcHPX15 clones are close orthologs of $A$. gambiae AgHPX15. The phylogenetic relationship of these anopheline HPX15 with other animal and plant heme peroxidases revealed that they form a separate lineage-specific cluster and their orthologs are not found in human, nematodes or other related arthropods such as, Drosophila, Aedes and Culex mosquitoes. However, their putative orthologs are present in 16 other globally distributed anophelines and exhibit a highly conserved amino acids identity in the range of $70-99 \%$. Based on these findings we propose that the anopheline-specific and evolutionary conserved heme peroxidase HPX15 may serve as a unique target for designing transmission-blocking strategies to block Plasmodium-mosquito cycle. These findings will generate new frontiers in the field of malaria research and disease control.

Keywords: Heme peroxidase; HPX15; Anopheles mosquito; Malaria transmission; Ortholog; Innate immunity; Plasmodium

Abbreviations: HPX: Heme Peroxidase; As: Anopheles stephensi; Ac: Anopheles culicifacies; Ag: Anopheles gambiae; WHO: World Health Organization

\section{Introduction}

Malaria is a major health problem in tropical and subtropical countries of the world. In spite of different control measures, approximately 3.2 billion people are at the risk of malaria throughout the world. WHO reported 198 million cases of malaria and 584,000 deaths world widely in 2013 [1]. In the South East Asian region, India alone contributes nearly 1.5 million cases of malaria [2]. Malaria is caused by Plasmodium (a protozoan), which is transmitted among humans by the female Anopheles mosquito. In India, there are approximately 58 anopheline mosquito species and amongst them, six are potent malaria vectors [3]. Anopheles culicifacies and A. stephensi are major vectors of rural and urban malaria, respectively [4].

Human race pays a heavy toll to malaria in terms of death and economic loss. This warrants an attention to develop novel effective methods that can control Plasmodium development either in human or mosquito host. Such strategies are synthesis of effective vaccine/drugs against Plasmodium and the application of synthetic mosquitocidal compounds [5]. Currently there is no licensed vaccine against malaria [6] and the drug resistance in Plasmodium is also a major challenge in this field [7]. Moreover, disease vectors developed resistance against many synthetic mosquitocidal compounds as well as their non-biodegradability is also a serious issue $[8,9]$. On the other hand, a potent method of controlling malaria may be impeding parasite development inside the mosquito through transmission blocking strategies. However, this requires exploring the molecular interactions of Plasmodium and mosquito immune system and identifying potent targets to manipulate mosquito immunity.

So far, major studies regarding Plasmodium-mosquito interactions are carried in African mosquito A. gambiae. In these mosquitoes numerous anti-bacterial and/or -plasmodial immune molecules have been identified, which effectively kill Plasmodium at different stages of development [10-17]. Some of these immune molecules successfully control the growth of Plasmodium berghei (mouse malaria) however, they are incompetent to regulate $P$. falciparum (human malaria) development $[18,19]$. These facts demand for the discovery of potent mosquito immune molecules that can broadly regulate the development of major human malaria parasites.

*Corresponding author: Sanjeev Kumar, Department of Biological Sciences, Birla Institute of Technology and Science (BITS), Pilani Campus, Pilani, Rajasthan India, Tel: + 911596515 670; E-mail: sanjeev@pilani.bits-pilani.ac.in

Received October 12, 2015; Accepted November 16, 2015; Published November 20, 2015

Citation: Kajla M, Gupta K, Kakani P, Dhawan R, Choudhury TP, et al. (2015) Identification of an Anopheles Lineage-Specific Unique Heme Peroxidase HPX15 A Plausible Candidate for Arresting Malaria Parasite Development. J Phylogen Evolution Biol 3: 160. doi:10.4172/2329-9002.1000160

Copyright: (C) 2015 Kajla M, et al. This is an open-access article distributed under the terms of the Creative Commons Attribution License, which permits unrestricted use, distribution, and reproduction in any medium, provided the original author and source are credited. 
Citation: Kajla M, Gupta K, Kakani P, Dhawan R, Choudhury TP, et al. (2015) Identification of an Anopheles Lineage-Specific Unique Heme Peroxidase HPX15: A Plausible Candidate for Arresting Malaria Parasite Development. J Phylogen Evolution Biol 3: 160. doi:10.4172/23299002.1000160

Recent studies identified A. gambiae heme peroxidases (HPXs) as major regulators of anti-P. falciparum immunity [20,21]. One of the $A$. gambiae heme peroxidase named AgHPX15 (AGAP013327) catalyzes protein cross-linking to form a physical barrier on the luminal surface of midgut epithelial cells. This barrier suppresses the recognition of blood bolus antigens by the mosquito immune system. The barrierbased mechanism maintains a 'low immunity' zone in this area to support the growth of endogenous bacteria in the blood fed midguts. Plasmodium takes the advantages of this situation to support its own development. Interestingly, the silencing of AgHPX15 gene suppressed the formation of midgut barrier and induced mosquito immunity against bacterial flora and P. facliparum [20]. Thus, AgHPX15 may be a 'potent candidate' that can be targeted to manipulate mosquito immunity to block Plasmodium development.

In this study, we characterized AgHPX15 orthologs in major Indian malaria vectors, A. stephensi and A. culicifacies and analyzed their evolutionary relationships. Our results demonstrate that HPX15 is an Anopheles lineage-specific gene and evolutionary conserved among nineteen different species of Anopheles mosquitoes. These findings may help us to design common strategies for blocking the transmission of human malaria by targeting a key central molecule that regulates Plasmodium development.

\section{Materials and Methods}

\section{Rearing of mosquitoes}

Anopheles stephensi mosquitoes were reared in insectory at $28^{\circ} \mathrm{C}$, $80 \%$ relative humidity $(\mathrm{RH})$ and $12 \mathrm{~h}$ light: dark cycle as described before [18]. Larvae were fed on a 1:1 mixture of dog food (Pet Lover's crunch milk biscuit, India) and fish food (Gold Tokyo, India). Adults were regularly maintained at $10 \%$ sucrose solution ad libitum. For colony propagation, adult females were fed on anesthetized mice and their eggs were collected in moist conditions. A. culicifacies has a complex of five sibling species named as A, B, C, D and E. All these sibling species, except $\mathrm{B}$, are good vectors for human malaria $(P$. falciparum and $P$. vivax) $[22,23]$. The sibling species A was reared in an insectory at Maharshi Dayanand University (MDU), Rohtak under the similar conditions as mentioned above. In addition, for rearing $A$. culicifacies the insectory was also equipped with simulated dusk and dawn system with a $14 \mathrm{~h}$ light and $10 \mathrm{~h}$ dark cycle as described before [24].

\section{Designing of $A$. stephensi heme peroxidase degenerate and gene-specific primers}

Initially at the starting of this study genome sequences were not available thus, degenerate primers approach was used to clone $A$. stephensi HPX15 (ortholog of A. gambiae AgHPX15). Degenerate primers were designed based on the conserved regions of different insect peroxidase proteins as illustrated in Figure S1. The sequences of degenerate primers ( 5 ' to $3^{\prime}$ ) were following:

\section{Forward (degF): TACTRBGARTGGYTGCCVATY,}

\section{Reverse (degR): GCCARWCCRTGRTCVCGRKYRCGCTG.}

Later on with the availability of A. stephensi genome (taxid: 30069), the AsHPX15 gene sequence was identified in contig 5285 (recently super contig KB665221, Ensembl gene identifier ASTE008179 in annotated genome) based on the nucleotide blast with the clone obtained by degenerate primers. The nucleotide sequence of this putative AsHPX15 gene was aligned with known nucleotide sequence of AgHPX15 to design gene-specific primers as illustrated in Figure S2 and their sequences (5' to 3') are following:

Forward 2 (F2): GAGAAGCTTCGCACGAGATTA,

Reverse 2 (R2): GAATGTCGATTGCTTTCAGGTC;

Forward 3 (F3): AGTGCAACAGCTTGCGTACC,

Reverse 3 (R3): CCTTTAGTCCATGAGTGTTGTCA.

\section{Mosquito tissue collection and RNA isolation}

A. stephensi and A.culicifacies females were allowed to feed on an anesthetized mouse. After $24 \mathrm{~h}$ of blood feeding the midgut $(\mathrm{Mg})$ and the rest of the body parts (carcass, CC) were collected from the pool of mosquitoes in RNAlater and stored at $-80^{\circ} \mathrm{C}$. Total RNA was isolated from these samples using RNAeasy mini kit from Qiagen (Cat no. 74104) following manufacturer's instructions.

\section{cDNA preparation and RT-PCR}

The first-strand cDNA was synthesized using Quantitech reverse transcription kit (Qiagen Cat no. 205311). RT-PCR was performed with midgut and carcass CDNA using above mentioned primers. PCR with degenerate primers followed the first cycle at $94^{\circ} \mathrm{C}$ for $3 \mathrm{~min}, 55^{\circ} \mathrm{C}$ for $1 \mathrm{~min}, 72^{\circ} \mathrm{C}$ for $3 \mathrm{~min}, 94^{\circ} \mathrm{C}$ for $1 \mathrm{~min}, 42^{\circ} \mathrm{C}$ for $1 \mathrm{~min}, 72^{\circ} \mathrm{C}$ for 3 min. Then another cycle at $94^{\circ} \mathrm{C}$ for $1 \mathrm{~min}, 52^{\circ} \mathrm{C}$ for $1 \mathrm{~min}, 72^{\circ} \mathrm{C}$ for 3 min was repeated 34 times with a final extension at $72^{\circ} \mathrm{C}$ for $10 \mathrm{~min}$.

\section{Cloning and sequencing of AsHPX15 and AcHPX15 heme peroxidases}

The resulting PCR product ( $428 \mathrm{bp}$, Figure 1A) with degenerate primers was cloned into PCR2.1-TOPO TA vector (Invitrogen). The clone was sequenced in an automated sequencer at the commercial sequencing facility. The sequence identity was confirmed through Mega blast against the nucleotide sequences database at NCBI. Further, this degenerate clone nucleotide sequence was blasted against $A$. stephensi genome to identify AsHPX15 putative gene as discussed above.

The primer set F3R3 amplified $\sim 1.1 \mathrm{~kb}$ PCR product from $A$. stephensi or A. culicifacies midgut CDNA templates and we termed them AsHPX15 and AcHPX15, respectively (Figure 1B and 1C). Further, the sequence identity of AsHPX15 and AcHPX15 was confirmed and submitted to NCBI. The sequence accession numbers [GenBank: KP223285] for A. stephensi AsHPX15 and [GenBank:KP299257] for A. culicifacies AcHPX15 were obtained. The primer set F2R2 was used to confirm the identity of all these clones through PCR (data not shown).

\section{Analysis of conserved domains in AsHPX15 and AcHPX15 sequences}

Signature domains in cloned AsHPX15 and AcHPX15 were identified using the Conserved Domain Database (CDD) search tool available online at NCBI [25]. These results were validated after comparing them to the CDD results of A. gambiae heme peroxidase AgHPX15 and other anopheline peroxidases.

\section{Selection of heme peroxidases for phylogenetic analysis}

The evolutionary relationship of cloned anopheline peroxidases (AsHPX15 and AcHPX15) was analyzed with selected 74 heme peroxidase protein sequences from various organisms (as shown in Table S1). These sequences were downloaded from NCBI and VectorBase databases and their overhanging sequences were trimmed based on predicted protein of AsHPX15 clone. Importantly, for these analyses, we selected heme peroxidase protein sequences from blood 
Citation: Kajla M, Gupta K, Kakani P, Dhawan R, Choudhury TP, et al. (2015) Identification of an Anopheles Lineage-Specific Unique Heme Peroxidase HPX15: A Plausible Candidate for Arresting Malaria Parasite Development. J Phylogen Evolution Biol 3: 160. doi:10.4172/23299002.1000160

Page 3 of 11

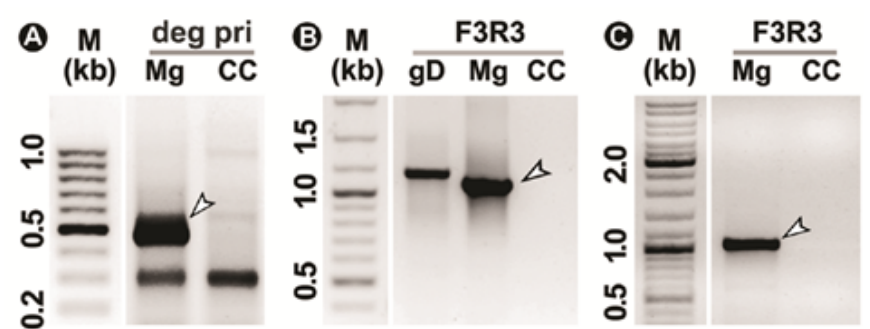

Figure 1: Cloning of HPX15 gene from Indian malaria vectors, $A$. stephensi and $A$. culicifacies.

A) $24 \mathrm{~h}$ post blood fed $A$. stephensi midgut $(\mathrm{Mg})$ and carcass (CC) cDNA templates were PCR amplifies using degenerate primers (deg pri) as depicted in Figure S1. These primers amplified a desired product of $428 \mathrm{bp}$ from the midgut (Mg) CDNA.

B) PCR amplification of $A$. stephensi midgut (Mg) and carcass (CC) cDNA using gene-specific primers (F3R3) as depicted in Figure S2. These primers amplified a single product of $\sim 1100$ bp exclusively from Mg cDNA. The genomic DNA (gD) template served as a control for the PCR reactions. C) A. culicifacies cDNA templates were PCR amplifies using F3R3 primers. The desired band of $\sim 1100$ bp is seen only in Mg cDNA. The leftmost lane in all the cases represents the standard DNA molecular marker $(M)$ reference for identifying the size of amplified DNA fragments. Numbers on the left side indicate standard DNA size in $\mathrm{kb}$. PCR products indicated by arrowheads were used for cloning and sequencing.

feeding (Pediculus humanus, Aedes aegypti, A. gambiae and Culex quinquefasciatus) as well as non blood feeding (Tribolium castaneum, Apis mellifera and Drosophila. melanogaster) arthropods. In addition, Caenorhabditis elegans, Arabidopsis thaliana and Homo sapiens heme peroxidases were also included to understand their phylogenetic relationship and advancements during evolution. In some analyses, we also included non heme peroxidase protein sequences from A. gambiae (Table S1)

To retrieve putative HPX15 peroxidases from additional 16 different anophelines, we blasted nucleotide sequence of AsHPX15 clone against their whole genome shotgun (WGS) sequences available at NCBI. The contig with best match was obtained and aligned with AsHPX15 clone by ClustalW algorithm to trim the overhanging regions. The predicted proteins for these trimmed sequences are mentioned in Table 1. Peroxidases from other organisms were also trimmed in the same way. The phylogenetic tree was constructed using the full length or trimmed protein sequences of these peroxidases. The tree topology was same for full length as well as trimmed sequences thus, the results with trimmed sequences are presented in this manuscript.

\section{Phylogenetic tree construction and analysis}

Phylogenetic trees were constructed from selected peroxidases using the maximum likelihood (ML) and neighbor-joining (NJ) methods implemented in MEGA 5.2 program as described before [26]. We aligned all selected protein sequences by ClustalW algorithm in the MEGA 5.2 program as before [27]. Following criteria were selected for the phylogenetic analysis: 'WAG' option was selected as the model for amino acid substitution as this model best fits to our data. For gaps and missing data, we used 'all sites' option. The ML tree was generated using nearest neighbor interchange (NNI) tree search algorithm. Branching pattern reliability was tested for both ML and NJ tree by 1000 bootstrap replicates. The resulting phylogenetic tree was analyzed based on clusters and nodes formed. Because the topologies of phylogenetic tree obtained by ML and NJ methods were similar, therefore, only the ML tree for respective analyses is presented in this study.

\section{Results}

\section{Characterization of AgHPX15 orthologs in A. stephensi and A. culicifacies}

To identify AgHPX15 orthologs in major Indian malaria vectors $(A$. stephensi and A. culicifacies), we cloned HPX15 from these mosquito species. Initially at the starting of this study A. stephensi genome sequences were not available thus, degenerate primers approach was used to clone HPX15 as discussed in Materials and Methods and Figure S1. A PCR product of $428 \mathrm{bp}$ from A. stephensi midgut cDNA was cloned and sequenced (Figure 1A). The nucleotide blast results revealed its closest match with A. gambiae AgHPX15 (77\% identity and E value 6e-84). This clone was named degAsHPX15.

Later on the unannotated genome of A. stephensi (taxid: 30069) was available at NCBI. The nucleotide blast of degAsHPX15 and AgHPX15 against A. stephensi genome retrieved contig 5285 (recently super contig KB665221, Ensembl identifier ASTE008179 in annotated genome), which was used to design gene-specific primers (Figure S2). PCR with F3R3 primers amplified a fragment of $\sim 1100$ bp exclusively from $A$. stephensi midgut cDNA and no product was observed when carcass cDNA was used as template (Figure 1B). The PCR with other primers F2R2 amplified a desired (329 bp) product from these clones (data not shown) and we used it to confirm the identity of our clones. We further continued with the larger $(\sim 1100 \mathrm{bp})$ PCR product obtained by F3R3 primers. This PCR product was sequenced (size 1075 bp), named as AsHPX15 and its sequence were submitted to NCBI [GenBank: KP223285]. The nucleotide or predicted protein blast of AsHPX15 identified its closest match to A. gambiae AgHPX15. NCBI nucleotide blast of obtained sequences disclosed $89 \%$ query coverage, 76\% identity and E value 3e-168 with A. gambiae HPX15 (AgHPX15).

Interestingly, the A. stephensi AsHPX15 specific F3R3 primers also amplified a similar size PCR product when A. culicifacies midgut cDNA was used as template (Figure 1C). The obtained clone was named as AcHPX15 and its sequence (1077 bp) was submitted to NCBI [GenBank: KP299257]. NCBI nucleotide blast of AcHPX15 sequences revealed first hit as AgHPX15 with 81\% query coverage, $77 \%$ identity and E value 0.0. Predicted proteins for AcHPX15 and AsHPX15 also have a close orthology to each other (79\% identity and $86 \%$ similarity). These results revealed that AgHPX15, AsHPX15 and AcHPX15 are identical orthologs.

\section{AgHPX15, AsHPX15 and AcHPX15 have identical domains}

To understand details regarding the sequence identity, structure and functional relationships, AgHPX15, AsHPX15 and AcHPX15 proteins were subjected to conserved domain database (CDD) analysis. Results depicted in Figure 2A revealed that all these peroxidases exhibit characteristics identity similar to the peroxinectin-like conserved domain of animal heme peroxidases superfamily. Peroxinectins are secreted as cell-adhesive and opsonic arthropod proteins that play crucial role in invertebrate immunity and interact with integrin family of transmembrane receptors [28]. Human myeloperoxidase (MPO) is also a member of this vast family and interacts with integrins $[29,30]$. Animal heme peroxidases and related proteins superfamily contains a diverse group of enzymes, including peroxidases from metazoans and their members are also found in fungi, plants and bacteria [31,32]. We also observed identical binding sites in the conserved domain of these three anopheline peroxidases. These sites are 10 heme binding, 14 putative substrate binding and 2 homodimer interface sites (Figure 2B). 
Citation: Kajla M, Gupta K, Kakani P, Dhawan R, Choudhury TP, et al. (2015) Identification of an Anopheles Lineage-Specific Unique Heme Peroxidase HPX15: A Plausible Candidate for Arresting Malaria Parasite Development. J Phylogen Evolution Biol 3: 160. doi:10.4172/23299002.1000160

Page 4 of 11

\begin{tabular}{|c|c|c|c|c|c|c|}
\hline $\begin{array}{l}\text { Vectorial } \\
\text { capacity }\end{array}$ & $\begin{array}{l}\text { Anopheles spp. } \\
\text { (abbreviation) }\end{array}$ & $\begin{array}{l}\text { Ensembl identifier or } \\
\text { gene bank identity }\end{array}$ & Retrieved contig & $\begin{array}{c}\% \text { nucleotide query } \\
\text { coverage with } \\
\text { AsHPX15 (KP223285) }\end{array}$ & $\begin{array}{l}\% \text { nucleotide Identity } \\
\text { with AsHPX15 } \\
\text { (KP223285) }\end{array}$ & $\begin{array}{l}\text { Nucleotides (Amino } \\
\text { acids) in trimmed } \\
\text { sequence }\end{array}$ \\
\hline \multirow[t]{12}{*}{ Major vectors } & A. arabiensis (Aar) & AARA008901 & 1.3480 & 96 & 75 & $1011(337)$ \\
\hline & A. atroparvus (Aat) & AATE013459 & 1.2435 & 90 & 70 & $934(311)$ \\
\hline & A. darling (Ada) & ADAC006384 & 7077 & 77 & 71 & $985(335)$ \\
\hline & A. dirus (Adi) & ADIR011596 & 1.4912 & 83 & 72 & $909(303)$ \\
\hline & A. farauti (Afa) & AFAF010327 & 2.1809 & 93 & 73 & $1015(337)$ \\
\hline & A. funestus (Afu) & AFUN008618 & 1.6688 & 98 & 76 & $995(331)$ \\
\hline & A. maculatus (Ama) & AMAM004556 & 1.2526 & 98 & 83 & $986(328)$ \\
\hline & A. sinensis (Asi) & Not known & 012260 & 96 & 69 & 1009 (336) \\
\hline & A. nili (Ani) & Not known & 19203 & 84 & 71 & $915(304)$ \\
\hline & A. gambiae (Ag) & AGAP013327 & - & 89 & 76 & $1005(335)$ \\
\hline & A. culicifacies (Ac) & KP299257 & 1.17233 & 84 & 79 & 1059 (353) \\
\hline & A. stephensi (As) & KP223285 & 5285 & 100 & 100 & $1075(358)$ \\
\hline \multirow[t]{5}{*}{ Minor vectors } & A. albimanus (Aal) & AALB010446 & 1.835 & 78 & 71 & $1050(323)$ \\
\hline & A. epiroticus (Aep) & AEPI000890 & 1.174 & 93 & 73 & $980(326)$ \\
\hline & A. melas (Amel) & AMEC000291 & 2.2419 & 96 & 74 & $995(331)$ \\
\hline & A. merus (Amer) & AMEM005191 & 2.5072 & 96 & 74 & $994(331)$ \\
\hline & A. minimus (Ami) & AMIN007008 & 1.3585 & 98 & 83 & $1009(336)$ \\
\hline \multirow[t]{2}{*}{ Non-vectors } & A. quadriannulatus (Aq) & AQUA003275 & 1.8077 & 96 & 75 & $1011(337)$ \\
\hline & A. christyi (Ach) & ACHR005516 & 1.2278 & 97 & 75 & $986(328)$ \\
\hline
\end{tabular}

Table 1: List of putative HPX15 peroxidases retrieved from different species of Anopheles mosquitoes.

The putative HPX15 peroxidase contig (or Ensembl identifier) from the genome of different anophelines were retrieved using nucleotide sequences of AsHPX15 clone (GenBank:KP223285) as query. The overhanging sequences of the contigs were trimmed based on AsHPX15 sequences as mentioned in the Materials and Methods. The blast query coverage, identity of corresponding HPX15 contig with AsHPX15 clone and the nucleotides and total amino acids in trimmed sequences are presented in the table. The abbreviation for individual mosquito is also mentioned in parenthesis.

\section{HPX15 is a unique anopheline-specific heme peroxidase}

The CDD analysis revealed that the domain structure of AgHPX15, AsHPX15 and AcHPX15 is identical and they all are designated heme peroxidases (Figure 2). In vertebrates and invertebrates heme peroxidases catalyze protein crosslinking, which is generally a crucial process to stabilize the extracellular matrix [20,31]. The basic mechanism of peroxidase-mediated protein crosslinking is evident in A. gambiae [20]. Thus, we believe that AsHPX15 and AcHPX15 may also demonstrate a similar mechanism in Indian malaria vectors. Therefore, these heme peroxidases were further analyzed to understand their common features.

As we discussed before that the nucleotide and predicted protein general blasts of AsHPX15 and AcHPX15 revealed A. gambiae AgHPX15 as closest match. However, they do not have a considerable match with any peroxidase from other organisms. Thus, we hypothesized that HPX15 is a unique type of heme peroxidase and its orthologs may be present only in the genus Anopheles. To understand the novelty of anopheline HPX15 peroxidases, we used WAG model to reconstruct their phylogenetic relationship with other peroxidases as explained in Material and Methods. For this analysis, we selected heme peroxidases from numerous blood feeding and non blood feeding insects, human and plant (as mentioned in Table S1). Results presented in Figure 3 revealed that these peroxidases appear in two major clades. Each clade defines separate lineage for plants and animal heme peroxidases. The clade for animal heme peroxidases is further divided into 11 subclades that are designated based on A. gambiae peroxidase representative (this nomenclature was adopted from VectorBase database) in the cluster of that particular subclade. For example, AgHPX4, AgHPX3, AgDBLOX, AgHPX6, AgHPX5, AgHPX7, AgHPX8, AgHPX1, AgHPX16 and AgDUOX (Figure 3).

Among 16 A. gambiae heme peroxidases, AgHPX4 and AgDUOX reveal orthology to a wide range of animal heme peroxidases that include human EPO and MPO and peroxidases from Caenorhabditis elegans, mosquitoes and other insects. This shows the ubiquitous nature of these genes that have been conserved during the evolution through lower to higher organisms. Interestingly, AgHPX3 orthologs are present only in arthropod as well as C. elegans. AgHPX1, AgHPX5, AgHPX6, AgHPX7 and AgDBLOX are arthropod-specific heme peroxidases (Figure 3) and might have conserved function across insect species irrespective of their blood feeding or non blood feeding behaviors as observed in case of other genes [33]. These peroxidases might have been selected by gene duplications that occurred prior to the most recent common ancestor (MRCA) of Hemimetabolous (e.g., Pediculus humanus, $\mathrm{Ph}$ ) and Holometabolous (e.g. mosquitoes) insects. AgHPX16 has no ortholog in other mosquitoes such as, Aedes and Culex however, its ortholog is present in Drosophila. AgHPX2 and AgHPX8 are mosquito-specific heme peroxidases and their orthologs are present in Aedes and Culex mosquitoes (Figure 3).

The above phylogenetic analysis also indicated the presence of lineage-specific mosquito heme peroxidases. Our findings revealed that there is a cluster of unique Anopheles heme peroxidases, which includes AgHPX10, AgHPX11, AgHPX12, AgHPX14 and AgHPX15. These A. gambiae-specific peroxidases do not have any ortholog in other arthropods that we analysed. Interestingly, AgHPX10 and AgHPX15 have their own paralogs named AgHPX11 and AgHPX14, respectively. However, the heme peroxidase AgHPX12 has no paralog even in $A$. gambiae (Figure 3). These unique peroxidases might have some crucial role in biology, physiology, geographical distribution or environmental adaptations of Anopheles mosquito as reported in case of other genes [33]. We emphasize that these peroxidases may be considered unique targets to synthesize blockers for regulating the biological activities of the malaria vector. However, the involvement of these peroxidases in the regulation of Plasmodium development needs further investigations and our group is actively engaged in that direction.

It is also evident from the phylogenetic analysis that AsHPX15, AcHPX15 and AgHPx15 are evolutionary closer orthologs (Figure 3). Thus, overall this data represents that HPX15 is a novel peroxidase 


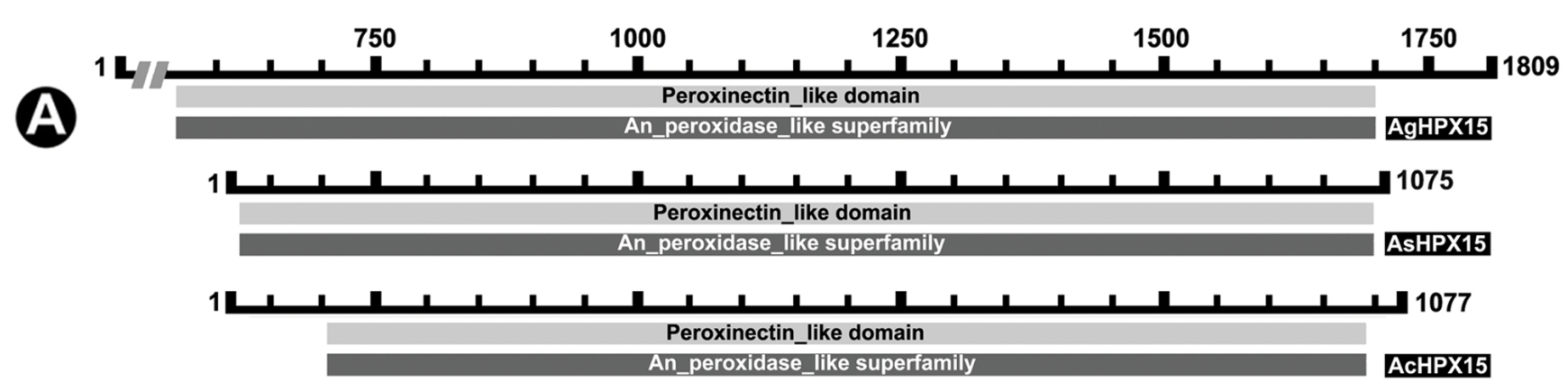

$\nabla \nabla$

AsHPX15

AcHPX15

AgHPX15

AsHPX15

AcHPX15

AgHPX15

AsHPX15

ACHPX15

AgHPX15

AsHPX15

AcHPX15

AgHPX15

AsHPX15

AcHPX15

AgHPX15

AsHPX15

AcHPX15

AgHPX15
-CNSLRTFEKGLLEVEHRNGKQWLPPHPNRTATCSVQDESE-SCYLTGDVRSNQSPHLTL -CNSLRTFTMGQMGVEQRNGKQWLPAHPNRTAVCNVKDDSE-ACYLTGDVRSNQSPHLTL QTNSLRTFSWGQLQAETRNGKQWLPVHPNKTTTCVSKDAADDACYLTGDVRSNQSPHLTL

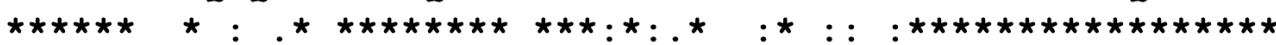

IHQAFVLEHNRLARELAELNAGWDDSVLFEQARKINIAQYQRIVYYEWLPIYLGRQNMLE IHQTFLLEHNRLARELATLYPDWDDNVLFQEARKINIAQYQRIVYYEWL PIYMGRQNMMA LHQAFHLEHNRLARELADLNAGWDDETVFQQARKLNIAQYQRIVYYEWLPIYLGAENMRA

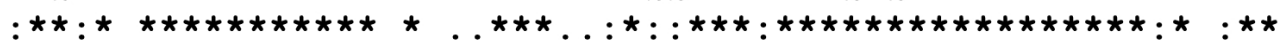

$$
\nabla \nabla \nabla \nabla
$$

TGVLPVLDVRSFARDYNHTVDPAVDNAFATAAFRFFHNLIAGHLDLVAESQQPTGSIRLS AGVLPELDERHFARDYNHTVDPTVDNAFATAAFRFFHNLIAGHLDLVAESKQPTGSIRLS AGVLPALELPGFADDYDASVDPTVSNAFATAAFRFFHNLIAGHLDLIAESRQPTGSIRLS

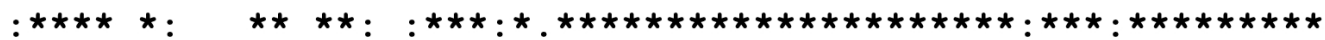
V $\eta$ DWFNKPSVLEMSDNYESLSRGMIYQPHDCPNNQLTPEVKHYLFRHGGRVGVDLKAIDIQR DWFNNPSVLEKDEYYGSLSRGMLYQPHDRPNCHLTPEVKHFLFRHGGAVGVDLKAIDIQR DWFNNPSVLEKDGNYEQLSRGMIYQPHDRPNHHLTPEVKHFLFRHGGPVGVDLKAIDIQR

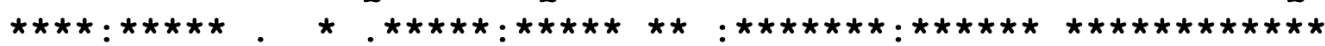
等 ARDHGLASYNDYREFCGLPRVTSWEELEDLLR-PDSARLLSQQYESVDDVDLAVGGALER ARDHGLGSYNDYREFCGLPRVSSWEGFIDLLS-PTSASSIRKHYESVDDVELAVAGALEG ARDHGLASYNDYREYCGLGRVTSWEEFNNLLRTPAMVRSLSEQYESVDDVDLAVAGALER

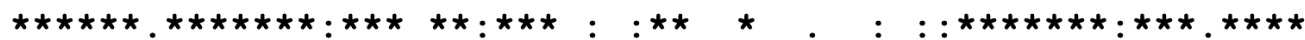

PYGDGMTGETFSCILLEQFRRTRVSDRFFFENDGQFTARQLLEVRKATMARVLCDNIHGL PYGDGMAGETFACILIDQFRRTRAGDRFYFENDSMFTQQQLHEIRKATMAKVLCDNTHGM HHGDGMPGETFACLLLDQFRRTRVGDRFYFENGNVFSSRQLFEVRKASMARVLCDNTHGL

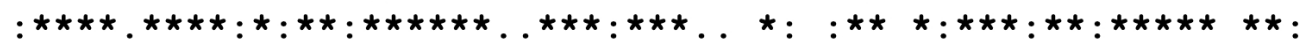

$\begin{array}{lll}\text { AsHPX15 } & \text { K- } & 358 \\ \text { AcHPX15 } & \text { E- } & 358 \\ \text { AgHPX15 } & \text { KE } & 572\end{array}$

$\nabla$ Heme binding site $\frac{m_{k}^{m}}{m_{m}}$ Homodimer interface $\square$ Putative substrate binding site

Figure 2: Analysis of conserve domains in AgHPX15, AsHPX15 and AcHPX15.

A) Signature domains in protein sequences of three heme peroxidases were determined through the Conserved Domain Database (CDD) search tool available online at NCBI. The number scale indicates amino acids in corresponding peroxidase proteins. Thick gray and black bars depict the peroxinectin-like conserved domain and animal heme peroxidases superfamily, respectively.

B) Numerous CDD predicted binding sites such as heme binding, putative substrate binding and homodimer interface sites are represented by the characteristic symbols in the aligned amino acids sequences of AgHPX15, AsHPX15 and AcHPX15. 


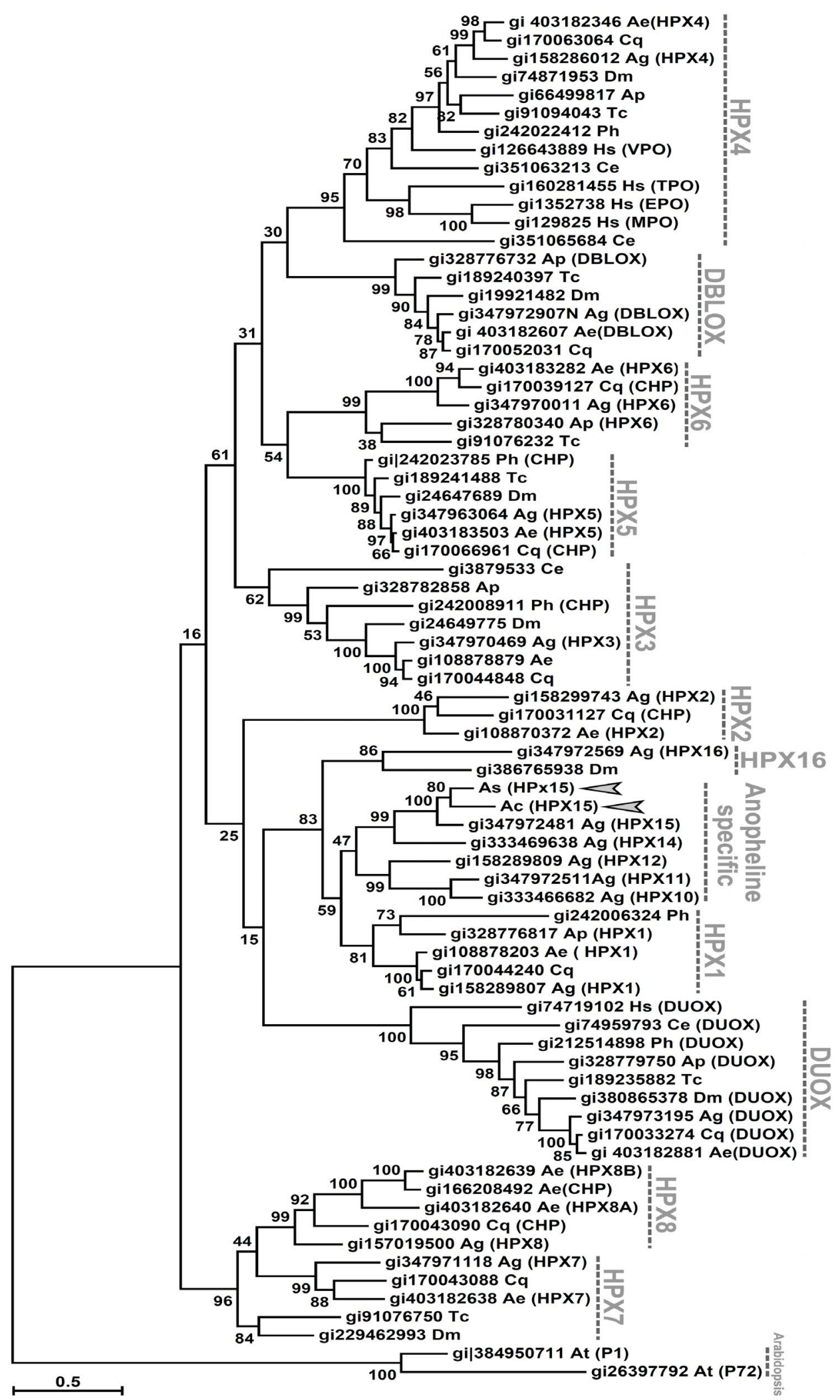

Figure 3: Phylogenetic analysis of selected heme peroxidases from various organisms

The Maximum likelihood (ML) method was used to construct the phylogenetic tree for 76 heme peroxidase (HPX) proteins selected from different organisms. The gene ID and abbreviations of organisms' scientific names have been mentioned in Table S1. Arrowheads indicate AsHPX15 and AcHPX15 clones. Vertical broken gray lines indicate specific clusters, which are named after the representative $A$. gambiae HPX. The scale bar represents base substitutions per site. The numbers on the branches represent the $\%$ of 1000 bootstrap. 
Citation: Kajla M, Gupta K, Kakani P, Dhawan R, Choudhury TP, et al. (2015) Identification of an Anopheles Lineage-Specific Unique Heme Peroxidase HPX15: A Plausible Candidate for Arresting Malaria Parasite Development. J Phylogen Evolution Biol 3: 160. doi:10.4172/23299002.1000160

Page 7 of 11

that is present at least in three different anopheline mosquitoes $(A$. gambiae, A. stephensi and A. culicifacies) and does not have ortholgs in any other dipterans. Further, we analyzed the phylogenetic relationship of AsHPX15 and AcHPX15 with all heme peroxidase proteins of related mosquitoes (all belonging to family Culicidae). For that, we selected total 42 HPX proteins from the genome of $A$. aegypti, C. quinquefaciatus and A. gambiae (as mentioned in Table S1). These sequences were trimmed as mentioned before in the Materials and Methods and reconstructed a phylogenetic tree using the WAG model (Figure 4). Interestingly, the topology of this phylogenetic relationship is broadly similar to that of the corresponding subclade in the previously constructed phylogenetic tree (comparing Figure 3 and Figure 4). This phylogenetic analysis clearly demonstrated that out of 16 A. gambiae heme peroxidases 10 have their orthologs in Aedes and Culex mosquitoes. These heme peroxidases are AgHPX1, AgHPX2, AgHPX3, AgHPX4, AgHPX5, AgHPX6, AgHPX7, AgHPX8, AgDUOX and AgDBLOX. This topology supports that these orthologs are selected by gene duplications that occurred prior to the most recent common ancestor (MRCA) of family Culicidae. Importantly, 5 heme peroxidases that further exhibited anophelines lineage specificity are AgHPX10, AgHPX11, AgHPX12, AgHPX14 and AgHPX15 (Figure 4). AgHPX16 seems to be the most divergent A. gambiae heme peroxidase and does not have any known paralog. However, we observed its ortholog in Drosophila (Figure 3). In addition, the anophelines heme peroxidases also experienced a lineage-specific expansion as reported in other insects [33]. The phylogenetic relationship among 16 A. gambiae heme peroxidases and 8 non heme peroxidases revealed that both types of peroxidases appear in two different clades and have diverged from each other a long before (Figure S3). It is also noteworthy to mention that gene expansion and duplication is dominant in A. gambiae heme peroxidases. For example, AgHPX15 and HPX14 are in same cluster (99\% bootstarp value), HPX12 is forming cluster with HPX10-HPX11 (98\% bootstarp value), HPX7-HPX8 and HPX4-HPX5 are also appearing in their own clusters, respectively (Figure S3). These clusters represent gene duplication (origin of paralogs) in heme peroxidases and explain the expansion of HPX family in A. gambiae. However, AgHPX1, AgHPX2, AgDUOX, AgHPX16 and AgDBLOX do not reveal any characteristic duplication pattern (Figure S3).

\section{HPX15 orthologs are present in the malaria vector and non vector species of Anopheles mosquitoes}

Our previous phylogenetic analyses revealed that HPX15 orthologs are present at least in three major malaria transmitting species of Anopheles mosquito such as, A. gambiae, A. stephensi and A. culicifacies (Figures 3 and 4 ). The recent availability of whole genome shotgun sequences of 16 additional species of anopheline mosquitoes drew our attention to understand the universality of HPX15 in these worldwidedistributed Anopheles mosquitoes. Nucleotide blast of AsHPX15 clone against these shotgun sequences provided us the matching contigs (recently named as ensemble identifier as mentioned in Table 1). We found that all of these 16 species of Anopheles mosquitoes also have the putative HPX15 gene in their genome. Interestingly, in this comprehensive analysis of 19 above-mentioned anophelines (see Table 1) there are twelve major, five minor and two non malaria vector species as defined by others [34]. Thus, the presence of HPX15-like peroxidases in the genome of vectors and non vector species may not reveal its direct association with their vectorial capacity. We believe that HPX15 might have a general physiological role in these blood feeding vectors or non vector species of Anopheles mosquitoes and managing the phenomenon of 'immune balance in midgut' during food digestion as we reported before in case of A. gambiae [20]. This further warrants the detailed investigations of these mechanisms in other anophelines.

\section{HPX15 proteins are highly conserved among anophelines}

We compared the predicted protein sequences of all the sixteen HPX15 peroxidases to analyze their relative identity with AgHPX15, AsHPX15 and AcHPX15 proteins. The amino acids identity among these total 19 HPX15 peroxidases was found to be highly conserved (70-99\%, Table 2). This range of percentage identity, confirmed that all these peroxidases are orthologs as described in case of other proteins [35].

In addition, we also analyzed the evolutionary relationship among these 19 species-specific peroxidase proteins. Results presented in Figure 5 revealed that the phylogenetic relationship of these nineteen HPX15 proteins follows a pattern similar to the evolutionary classification of these mosquito species as described by other researchers (Figure 1 of [34]). These findings explain that HPX15 is conserved among anopheline mosquitoes and might have evolved the same way as different species of Anopheles evolved. In parallel, the nucleotide based phylogenetic tree of these 19 peroxidases (using Tamura Nei model) also revealed similar branching pattern (data not shown).

In conclusion, HPX15 is a highly conserved anopheline lineagespecific peroxidase and present in the genome of globally distributed major/minor malaria vectors. These findings have great advantage to the society in terms of blocking the activity of this single molecule might interrupt the malaria cycle in those vectors.

\section{Discussion}

Heme peroxidases (HPXs) are found almost in all living organisms and generally catalyze the one- and two-electron oxidation of numerous organic and inorganic substrates. The redox cofactor is heme b or post translationally modified heme that is ligated to either histidine or cysteine residue. Four heme peroxidase superfamilies (peroxidasecatalase, peroxidase-cyclooxygenase, peroxidase-chlorite dismutase and peroxidase-peroxygenase) have differences in their enzymatic activities and also evolved independently [36].

In general, heme peroxidases play an important role in development and immunity. In vertebrates and invertebrates, heme peroxidases catalyze protein crosslinking that is a crucial process to stabilize the extracellular matrix. This crosslinking is a fundamental feature of basal membranes and essential as an elemental mechanism of tissue biogenesis $[37,38]$. Heme peroxidases are also extensively involved in the evolution and adaptations to the environment [33]. Thus, HPXs superfamily is considered to be a group of highly diversified members. Our phylogenetic analysis of the heme peroxidases (HPXs) from different phyla revealed the existence of two separate lineages of highly diversified HPXs, namely animals and plants (Figure 3). These results are supported by the previous reports where heme peroxidases are classified into two major families, namely the animal and non-animal peroxidases that include fungal and protist heme peroxidases [32].

Our phylogenetic analysis of 74 animal heme peroxidases (16 from A. gambiae and 58 from other animals) reveal that they appear in 11 different clades. Some of the A. gambiae heme peroxidases have their orthologs in other organisms, including human (Figure 3). For example, an extensively conserved HPX4 and DUOX clusters, which are originated by gene duplication events that occurred during the evolution of the kingdom Animalia. In addition, some of the $A$. gambiae heme peroxidases (e.g., HPX1, HPX3 and HPX5) are more close relatives of other arthropods and emerged by gene duplications that occurred prior to the most recent common ancestor (MRCA) of hemimetabola and holometabola in the late carboniferous period, 


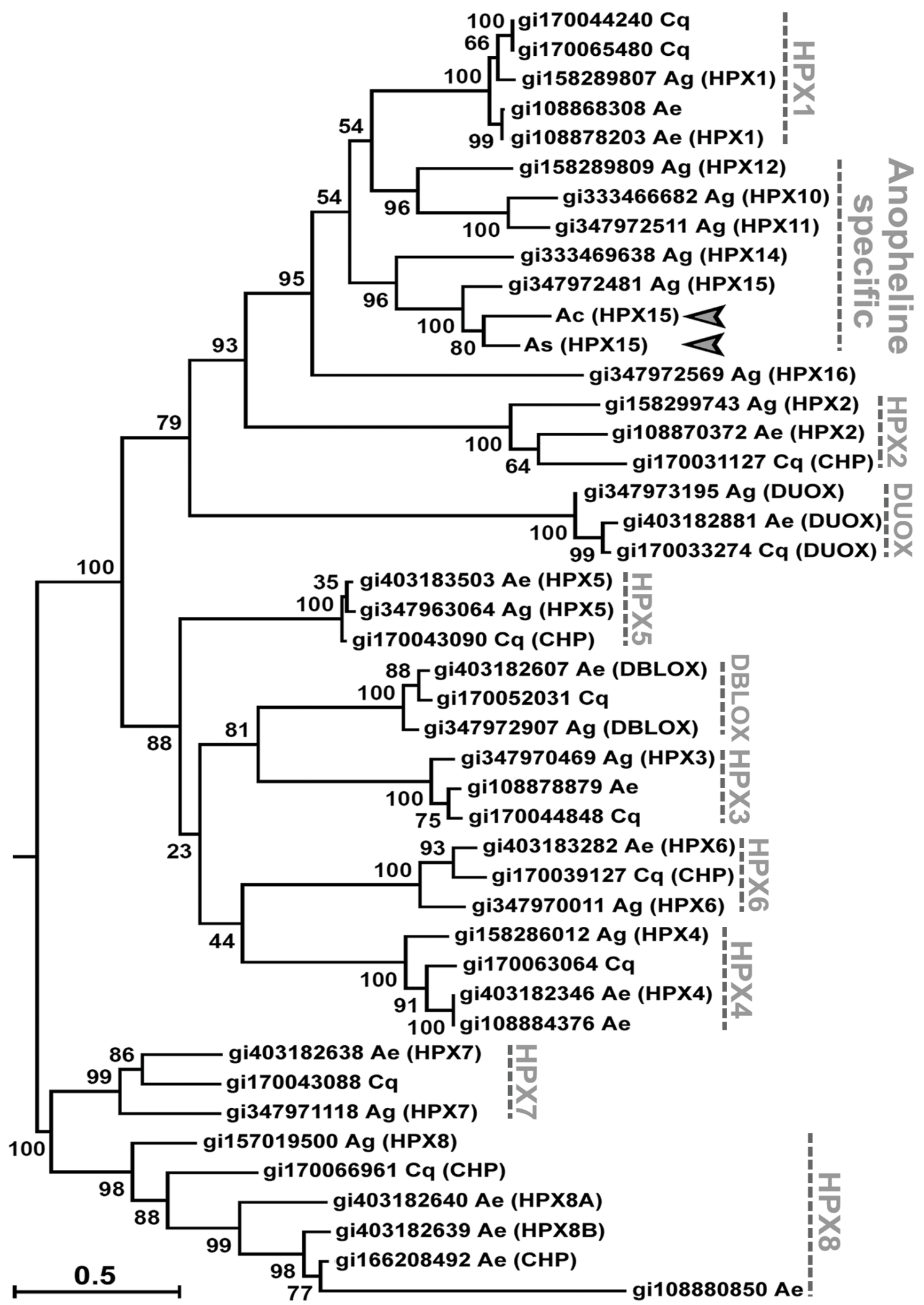

Figure 4: Phylogenetic analysis of mosquito's heme peroxidases. The ML method was used to construct the phylogenetic tree of 44 heme peroxidases selected from Aedes aegypti, Culex quinquefaciatus and Anopheles gambiae as discussed in Materials and Methods. The gene ID and organisms details are mentioned in Table $\mathrm{S} 1$. Arrowheads indicate AsHPX15 and AcHPX15 clones. Vertical broken gray lines indicate specific clusters, which are named after the representative A. gambiae HPX. The scale bar represents base substitutions per site. The numbers on the branches represent the $\%$ of 1000 bootstrap. 
Citation: Kajla M, Gupta K, Kakani P, Dhawan R, Choudhury TP, et al. (2015) Identification of an Anopheles Lineage-Specific Unique Heme Peroxidase HPX15: A Plausible Candidate for Arresting Malaria Parasite Development. J Phylogen Evolution Biol 3: 160. doi:10.4172/23299002.1000160

Page 9 of 11

\begin{tabular}{|c|c|c|c|c|c|c|c|c|c|c|c|c|c|c|c|c|c|c|c|c|c|}
\hline & & AA & 1 & 2 & 3 & 4 & 5 & 6 & 7 & 8 & 9 & 10 & 11 & 12 & 13 & 14 & 15 & 16 & 17 & 18 & 19 \\
\hline 1 & AarHPX15 & 337 & & 98.81 & 99.10 & 97.58 & 97.28 & 84.76 & 72.46 & 69.97 & 4.28 & 72.54 & 74.67 & 77.89 & 76.05 & 72.24 & 9.04 & 77.34 & 8.96 & 6.88 & 5.46 \\
\hline 2 & $\mathrm{AqH}$ & 337 & 98.81 & & 99.10 & 97.58 & 97.28 & 84.76 & 3.19 & 70.27 & 4.28 & 1.94 & 74.01 & 77.56 & 6.35 & 71.64 & 9.34 & 77.64 & 8.66 & 6.58 & 4.54 \\
\hline 3 & A & 335 & .10 & 9.10 & & 97.89 & 97.58 & 85.37 & 3.55 & 70.87 & .92 & .37 & .01 & 77.56 & 7.11 & 72.97 & 0.18 & 78.25 & 9.57 & 7.48 & 4.85 \\
\hline 4 & 15 & 3 & .58 & .58 & 97.89 & & 8.68 & 84.36 & 2.46 & 69.91 & 3.63 & 1.73 & 73.36 & 77.56 & 76.83 & 72.95 & 9.64 & 77.51 & 78.96 & 7.20 & 4.54 \\
\hline 5 & 5 & 3 & .28 & .28 & 97.58 & .68 & & 85.89 & 2.83 & 70.21 & נ..5. & 2.04 & 74.01 & 77.23 & 7.13 & 73.25 & 0.55 & 78.72 & 9.27 & 8.12 & 4.85 \\
\hline 6 & AchHPX15 & 20 & 6 & 76 & 8 & 4.36 & 85.89 & & 5.82 & 72.87 & U.J2 & 73.78 & 73.42 & 76.33 & 7.06 & 74.09 & 80.18 & 80.79 & 79.38 & 22 & 9.57 \\
\hline 7 & alHPX15 & 3 & 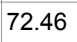 & 73.19 & 5 & 6 & 7 & 75.82 & & 4 & 4.28 & 4.28 & 70.65 & 71.64 & 4.64 & 71.01 & 2.83 & 72.83 & 1.38 & 69.20 & 7.03 \\
\hline 8 & daHPX15 & ; & .97 & .2 & 7 & 9.91 & 70.21 & 72.87 & 1.34 & & 73.95 & 72.97 & 70.72 & 72.28 & 72.59 & 65.77 & 70.57 & 71.00 & 69.51 & 67.87 & 66.26 \\
\hline 9 & 10 & 311 & .28 & .20 & 7 & 73.63 & 73.95 & 75.32 & 4.28 & 73.95 & & 87.46 & 77.96 & 76.79 & 76.45 & 70.42 & 74.60 & 73.31 & 73.31 & 72.67 & 67.85 \\
\hline 10 & AsiH & 33 & .54 & .94 & 7 & .73 & .04 & 8 & 28 & 72.97 & 87.46 & & 76.97 & 77.23 & 75.22 & 66.67 & 2.54 & 72.21 & 73.48 & 1.77 & 7.18 \\
\hline 11 & AniH & 304 & 67 & .0 & 7 & 3.36 & 01 & 2 & .65 & 70.72 & .96 & 76.97 & & 77.85 & 6.32 & 71.71 & 2.37 & 73.03 & 2.70 & 72.37 & 0.07 \\
\hline 12 & AdiHPX15 & 303 & .89 & 7.56 & 77.56 & 77.56 & 77.23 & 76.33 & 1.64 & 72.28 & 76.79 & 77.23 & 77.85 & & 5.15 & 76.24 & 7.89 & 76.90 & 77.56 & 73.60 & 71.62 \\
\hline 13 & AfaH & 337 & .05 & 76.35 & 77.11 & 76.83 & 77.13 & .06 & .64 & 72.59 & 76.45 & 75.22 & 76.32 & 85.15 & & 94 & 76.42 & 76.36 & 75.54 & 74.40 & 71.08 \\
\hline 14 & AcHPX15 & 337 & 72.24 & 1.64 & 72.97 & 72.95 & 73.25 & 74.09 & 71.01 & 65.77 & 70.42 & 66.67 & 71.71 & 76.24 & 69.94 & & 85.12 & 79.76 & 81.40 & 75.08 & 71.78 \\
\hline 15 & AmiHPX15 & 336 & 79.04 & 79.34 & 80.18 & 79.64 & 80.55 & 80.18 & 2.83 & 70.57 & 74.60 & 72.54 & 72.37 & 77.89 & 76.42 & 85.12 & & 86.71 & 85.98 & 81.08 & 73.31 \\
\hline 16 & AfuHPX15 & 331 & 77.34 & 77.64 & 78.25 & 77.51 & 78.72 & 80.79 & 72.83 & 71.00 & 73.31 & 72.21 & 73.03 & 76.90 & 76.36 & 79.76 & 86.71 & & 82.62 & 80.66 & 72.70 \\
\hline 17 & AmaHPX15 & 336 & 78.96 & 78.66 & 79.57 & 78.96 & 79.27 & 79.38 & 71.38 & 69.51 & 73.31 & 73.48 & 72.70 & 77.56 & 75.54 & 81.40 & 85.98 & 82.62 & & 87.50 & 73.01 \\
\hline 18 & AsHPX15 & 333 & 76.88 & 76.58 & 77.48 & 77.20 & 78.12 & 76.22 & 69.20 & 67.87 & 72.67 & 71.77 & 72.37 & 73.60 & 74.40 & 75.08 & 81.08 & 80.66 & 87.50 & & 70.25 \\
\hline 19 & AepHPX15 & 326 & 75.46 & 74.54 & 74.85 & 74.54 & 74.85 & 79.57 & 57.03 & 66.26 & 67.85 & 67.18 & 70.07 & 71.62 & 71.08 & 71.78 & 73.31 & 72.70 & 73.01 & 70.25 & \\
\hline
\end{tabular}

Table 2: Percentage amino acids identity among HPX15 peroxidases from nineteen different anophelines. The percentage identity among 19 different HPX15 peroxidase proteins was analyzed through their ClustalW alignments. The total amino acids (AA) used for analysis and the name of individual peroxidases (1 to 19) are also mentioned in the table. The protein sequences, gene ID and abbreviations of anophelines' scientific names are same as mentioned in Table 1.

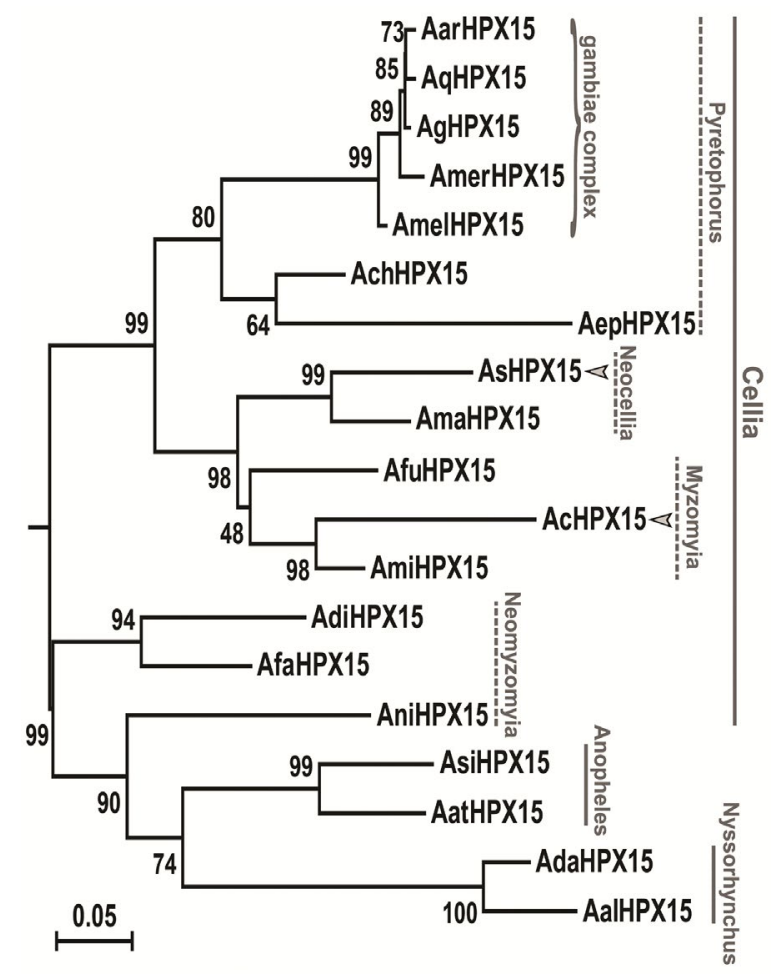

Figure 5: Phylogenetic analysis of putative HPX15 peroxidases from different species of Anopheles mosquitoes.

$\mathrm{ML}$ tree was constructed using heme peroxidase protein sequences from 19 different species of Anopheles mosquitoes. Details regarding HPXs nomenclature and anopheline species are mentioned in Table 1. Arrowheads indicate AsHPX15 and AcHPX15. Gray color solid vertical lines represent three sub genera (Cellia, Anopheles and Nyssorhynchus) of the genus Anopheles. Broken vertical lines represent series (Neocellia, Myzomyia, Neomyzomyia and Pyretophorus) of sub genus Cellia. The gambiae complex is the part of series Pyretophorus. The scale bar represents base substitutions per site. The numbers on the branches represent the $\%$ of 1000 bootstrap. 
Citation: Kajla M, Gupta K, Kakani P, Dhawan R, Choudhury TP, et al. (2015) Identification of an Anopheles Lineage-Specific Unique Heme Peroxidase HPX15: A Plausible Candidate for Arresting Malaria Parasite Development. J Phylogen Evolution Biol 3: 160. doi:10.4172/23299002.1000160

Page 10 of 1

318-300 million years ago [39,40]. Thus, it is clearly evident that gene duplication event in heme peroxidase superfamily is crucial in the evolution of biological complexity and offers raw material that diverged under positive selection [41]. These events are critical during evolution, speciation and the birth of new life forms.

In A. gambiae there are 16 heme and 8 non heme peroxidases, which are evolutionary separated from each other (Figure S3). This indicates that in A. gambiae there is a large expansion of heme peroxidases and that may be associated with their geographical distribution or biological behaviors as reported in other organisms [33]. It is also noticeable that some lineage-specific heme peroxidses are present only in anophelines and do not have any ortholog in other animals including Aedes and Culex mosquitoes. These unique Anopheles heme peroxidases are HPX10, HPX11, HPX12, HPX14 and HPX15 (Figure 3). In this group, HPX12 has no paralog in A. gambiae. However, HPX10-HPX11 and HPX14-HPX15 are paralogs.

As we have mentioned before that heme peroxidases are important to perform numerous biological functions in living organisms including insects. Anopheles mosquito is a known vector for human malaria therefore; we were interested to identify those novel heme peroxidases in this insect that regulate Plasmodium development. Among these above-mentioned unique A. gambiae peroxidases, AgHPX15 demonstrated a general physiological role in the blood fed mosquitoes, which indirectly supports Plasmodium development [20]. Thus, we believe that AgHPX15 might be considered a potent target to block the malaria cycle in A. gambiae.

The advocacy of AgHPX15 as a potent candidate for blocking malaria parasite development in mosquito requires that two important issues should be addressed. Whether, HPX15 is also present in all the known species of Anopheles, which are considered to be major/minor malaria vectors. So this single molecule may be a central point of interest to target all these vector species. Secondly, how conserved this molecule is so that a common approach to block its activity will be enough to control Plasmodium development in all worldwide-distributed malaria vectors. To address the first issue, we provided evidences that AgHPX15 orthologs are present in major Indian malaria vectors, A. stephensi and A. culicifacies (Figure 1, 3 and 4). In addition, our phylogenetic analysis with the retrieved sequences from 16 additional vector as well as nonvector anophelines also proved that the putative HPX15 gene is present in all these species (Figure 5). It is interesting to note that HPX15 is present in both vector and non vector anophelines. This may be due to the reason that all these mosquitoes are hematophagous in nature and thus, HPX15 may be required to crosslink the midgut barrier around the ingested blood in a way similar to A. gambiae [20]. However, it warrants further analysis and understanding the correlation between the HPX15 expression and blood feeding in these mosquito species individually.

For developing blocking strategies, the polymorphism in the target molecules is also a hurdle as reported in other systems [42]. Thus, it is important to understand the genetic diversity in HPX15 orthologs as a general rule. The conserved domain (CD) analysis of AgHPX15, AsHPX15 and AcHPX15 provided the details about their identity and functional relationships (Figure 2). Moreover, we also found the same conserved domain architectures when we analyzed other 16 anopheline putative HPX15 protein sequences (data not shown). A further comparison of amino acids among total 19 HPX15 peroxidases also revealed 70-99\% identity (Table 2). These observations strengthen our hypothesis that HPX15 can be a central plausible target and a common strategy may be applied to block its functioning to regulate the vectorial capacity of worldwide-distributed malaria vectors. Our previous double stranded (ds) RNA-mediated gene silencing experiments add in proof to this concept. Here the dsAgHPX15 RNA, prepared from A. gambiae cDNA, could silence HPX15 gene in both A. gambiae and A. stephensi. Moreover, the silencing-mediated effects on Plasmodium development were same in these mosquitoes (Figures 2 and S6 of [20]. These effects were probably due to the high $(\sim 75 \%)$ sequence identity between AgHPX15 and AsHPX15, which is evident now from the present study.

Recently a remarkable role of A. gambiae AgHPX15 gene is also reported in a study. Here this research group demonstrated that AgHPX15 induction in female spermathica is regulated by sexually transferred 20-hydroxyecdysone (20E), which is essential to preserve the functionality of stored sperm and long-term fertility [43]. Thus, targeting AgHPX15 will disrupt the reproductive cycle and numbers of mosquitoes in the field. These findings indicate that blocking the function of HPX15 will be beneficial in many ways and can be easily achieved as reported previously for other mosquito targets [44,45]. In these studies, a midgut-specific monoclonal antibody demonstrated a dose-dependent blocking effect against $P$. yoelii development in $A$. stephensi. Thus, we propose that the evolutionary conserved HPX15 protein in several anopheline mosquitoes can also be targeted in a similar way and may certify this molecule as a unique candidate to block mosquito cycle of Plasmodium development.

\section{Conclusion}

A lineage-specific heme peroxidase HPX15 is present in several globally distributed species of Anopheles mosquito. The highly conserved nature of HPX15 reveals that this molecule can be a potent target for blocking Plasmodium development. These findings may be a great help to fight against malaria, one of the world's deadliest diseases.

\section{Acknowledgement}

The authors wish to thank the laboratory staff for maintaining the insectory and central animal facilities (CAF) for providing all the supports. Special thanks to Divya Vaswani for providing critical comments. MK and KG are recipients of Basic Science Research (BSR) fellowship from the University Grants Commission (UGC) India. This work was supported by grants from the Department of Science and Technology (DST), Government of India (grant number SR/SO/HS-0131/2010).

\section{References}

1. World Health Organization. World malaria report (2014) Geneva: World Health Organization

2. National Vector Borne Disease Control Program (2013) National Drug Policy NVBDCP, India.

3. Nagpal BN, Sharma VP (1995) Indian anophelines. New Delhi: Oxford \& IBH Publishing Co. Pvt. LTD.

4. Subbarao SK (1998) Anopheline species complexes in South-East Asia. World Health Org Tech Pub 18: 82.

5. Shiff C (2002) Integrated approach to malaria control. Clin Microbiol Rev 15 278-293.

6. Crompton PD, Moebius J, Portugal S, Waisberg M, Hart G, et al. (2014) Malaria immunity in man and mosquito: insights into unsolved mysteries of a deadly infectious disease. Annu Rev Immunol 32: 157-187.

7. Klein EY (2013) Antimalarial drug resistance: a review of the biology and strategies to delay emergence and spread. Int J Antimicrob Agents 41: 311317

8. Kelly-Hope L, Ranson H, Hemingway J (2008) Lessons from the past: managing insecticide resistance in malaria control and eradication programmes. Lancet Infect Dis 8: 387-389.

9. Mrema EJ, Rubino FM, Brambilla G, Moretto A, Tsatsakis AM, et al. (2013) Persistent organochlorinated pesticides and mechanisms of their toxicity. 
Citation: Kajla M, Gupta K, Kakani P, Dhawan R, Choudhury TP, et al. (2015) Identification of an Anopheles Lineage-Specific Unique Heme Peroxidase HPX15: A Plausible Candidate for Arresting Malaria Parasite Development. J Phylogen Evolution Biol 3: 160. doi:10.4172/23299002.1000160

Page 11 of 11

Toxicology 307: 74-88.

10. Sreenivasamurthy SK, Dey G, Ramu M, Kumar M, Gupta MK, et al. (2013) A compendium of molecules involved in vector-pathogen interactions pertaining to malaria. Malar J 12: 216

11. Barillas-Mury C, Kumar S (2005) Plasmodium-mosquito interactions: a tale of dangerous liaisons. Cell Microbiol 7: 1539-1545.

12. Dimopoulos G, Christophides GK, Meister S, Schultz J, White KP, et al. (2002) Genome expression analysis of Anopheles gambiae: responses to injury, bacterial challenge, and malaria infection. Proc Natl Acad Sci U S A 99: 88148819.

13. Luckhart S, Li K, Dunton R, Lewis EE, Crampton AL, et al. (2003) Anopheles gambiae immune gene variants associated with natural Plasmodium infection. Mol Biochem Parasitol 128: 83-86.

14. Rivero A (2006) Nitric oxide: an antiparasitic molecule of invertebrates. Trends Parasitol 22: 219-225.

15. Gupta L, Molina-Cruz A, Kumar S, Rodrigues J, Dixit R, et al. (2009) The STAT pathway mediates late-phase immunity against Plasmodium in the mosquito Anopheles gambiae. Cell Host Microbe 5: 498-507.

16. Meister S, Kanzok SM, Zheng XL, Luna C, Li TR, et al. (2005) Immune signaling pathways regulating bacterial and malaria parasite infection of the mosquito Anopheles gambiae. Proc Natl Acad Sci U S A 102: 11420-11425.

17. Povelones M, Waterhouse RM, Kafatos FC, Christophides GK (2009) Leucinerich repeat protein complex activates mosquito complement in defense against Plasmodium parasites. Science 324: 258-261.

18. Jaramillo-Gutierrez G, Rodrigues J, Ndikuyeze G, Povelones M, MolinaCruz A, et al. (2009) Mosquito immune responses and compatibility between Plasmodium parasites and anopheline mosquitoes. BMC Microbiol 9: 154.

19. Molina-Cruz A, DeJong RJ, Ortega C, Haile A, Abban E, et al. (2012) Some strains of Plasmodium falciparum, a human malaria parasite, evade the complement-like system of Anopheles gambiae mosquitoes. Proc Natl Acad Sci U S A 109: E1957-1962.

20. Kumar S, Molina-Cruz A, Gupta L, Rodrigues J, Barillas-Mury C (2010) A peroxidase/dual oxidase system modulates midgut epithelial immunity in Anopheles gambiae. Science 327: 1644-1648.

21. Oliveira Gde A, Lieberman J, Barillas-Mury C (2012) Epithelial nitration by a peroxidase/NOX5 system mediates mosquito antiplasmodial immunity. Science 335: 856-859.

22. Barik TK, Sahu B, Swain V (2009) A review on Anopheles culicifacies: from bionomics to control with special reference to Indian subcontinent. Acta Trop 109: 87-97.

23. Subbarao SK, Adak T, Vasnatha K, Joshi H, Raghavandra K, et al. (1988) Susceptibility of Anopheles culicifacies species A and B to Plasmodium vivax and Plasmodium falciparum as determined by immunoradiometric assay. Trans Royal Soc Trop Med Hyg 82: 394-397.

24. Adak T, Kaur S, Singh OP (1999) Comparative susceptibility of different members of the Anopheles culicifacies complex to Plasmodium vivax. Trans $\mathrm{R}$ Soc Trop Med Hyg 93: 573-577.

25. Marchler-Bauer A, Derbyshire MK, Gonzales NR, Lu S, Chitsaz F, et al. (2015) CDD: NCBl's conserved domain database. Nucleic Acids Res 43: D222-226.

26. Saitou N, Nei M (1987) The neighbor-joining method: a new method for reconstructing phylogenetic trees. Mol Biol Evol 4: 406-425.

27. Tamura K, Peterson D, Peterson N, Stecher G, Nei M, et al. (2011) MEGA5 molecular evolutionary genetics analysis using maximum likelihood evolutionary distance, and maximum parsimony methods. Mol Biol Evol 28:
2731-2739.

28. Sritunyalucksana K, Wongsuebsantati K, Johansson MW, Söderhäll K (2001) Peroxinectin, a cell adhesive protein associated with the proPO system from the black tiger shrimp, Penaeus monodon. Dev Comp Immunol 25: 353-363.

29. Hampton MB, Kettle AJ, Winterbourn CC (1996) Involvement of superoxide and myeloperoxidase in oxygen-dependent killing of Staphylococcus aureus by neutrophils. Infect Immun 64: 3512-3517.

30. Persson T, Andersson P, Bodelsson M, Laurell M, Malm J, et al. (2001) Bactericidal activity of human eosinophilic granulocytes against Escherichia coli. Infect Immun 69: 3591-3596.

31. Dunford HB (1999) Heme peroxidases. New York: John Wiley and Sons Inc.

32. Passardi F, Bakalovic N, Teixeira FK, Margis-Pinheiro M, Penel C, et al. (2007) Prokaryotic origins of the non-animal peroxidase superfamily and organellemediated transmission to eukaryotes. Genomics 89: 567-579.

33. Shi GQ, Yu QY, Zhang Z (2012) Annotation and evolution of the antioxidan genes in the silkworm, Bombyx mori. Arch Insect Biochem Physiol 79: 87-103.

34. Neafsey DE, Christophides GK, Collins FH, Emrich SJ, Fontaine MC, et al (2013) The evolution of the Anopheles 16 genomes project. G3 (Bethesda) 3 1191-1194.

35. Peterson ME, Chen F, Saven JG, Roos DS, Babbitt PC, et al. (2009) Evolutionary constraints on structural similarity in orthologs and paralogs. Protein Sci 18: 1306-1315

36. Zámocký M, Hofbauer S, Schaffner I, Gasselhuber B, Nicolussi A, et al. (2015) Independent evolution of four heme peroxidase superfamilies. Arch Biochem Biophys 574: 108-119.

37. Péterfi Z, Geiszt M (2014) Peroxidasins: novel players in tissue genesis. Trends Biochem Sci 39: 305-307.

38. Kumar S, Barillas-Mury C (2005) Ookinete-induced midgut peroxidases detonate the time bomb in anopheline mosquitoes. Insect Biochem Mol Biol 35: 721-727.

39. Wiegmann BM, Trautwein MD, Kim JW, Cassel BK, Bertone MA, et al. (2009) Single-copy nuclear genes resolve the phylogeny of the holometabolous insects. BMC Biol 7: 34-47.

40. Hughes AL (2012) Evolution of the heme peroxisases of Culicidae (Diptera). Psyche 146387

41. Ohno S (1970) Evolution by gene duplication. New York: Springer-Verlag

42. Mahajan RC, Farooq U, Dubey ML, Malla N (2005) Genetic polymorphism in Plasmodium falciparum vaccine candidate antigens. Indian J Pathol Microbiol 48: $429-438$

43. Shaw WR, Teodori E, Mitchell SN, Baldini F, Gabrieli P, et al. (2014) Mating activates the heme peroxidase HPX15 in the sperm storage organ to ensure fertility in Anopheles gambiae. Proc Natl Acad Sci U S A 111: 5854-5859.

44. Dinglasan RR, Fields I, Shahabuddin M, Azad AF, Sacci JB Jr (2003) Monoclonal antibody MG96 completely blocks Plasmodium yoelii development in Anopheles stephensi. Infect Immun 71: 6995-7001.

45. Shahabuddin M, Cociancich S, Zieler H (1998) The Search for Novel Malaria Transmission-blocking Targets in the Mosquito Midgut. Parasitol Today 14 493-497. 